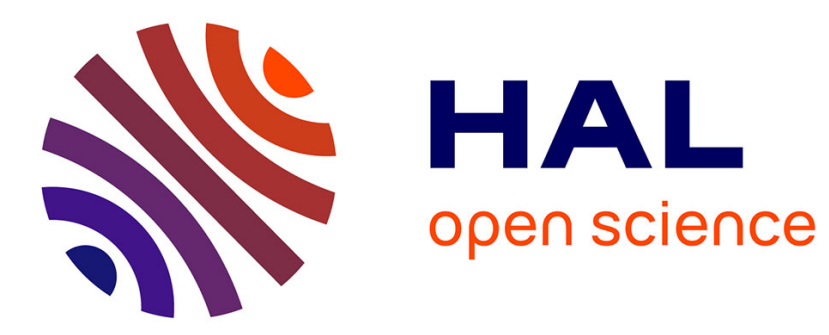

\title{
Optimal Capacity Allocation for a Failure Resilient Electrical Infrastructure
}

Yi-Ping Fang, Nicola Pedroni, Enrico Zio

\section{To cite this version:}

Yi-Ping Fang, Nicola Pedroni, Enrico Zio. Optimal Capacity Allocation for a Failure Resilient Electrical Infrastructure. DSM 2014, Jul 2014, Châtenay-Malabry, France. pp.197 - 207, 10.3139/9781569904923.020 . hal-01108228

\section{HAL Id: hal-01108228}

https://hal-centralesupelec.archives-ouvertes.fr/hal-01108228

Submitted on 22 Jan 2015

HAL is a multi-disciplinary open access archive for the deposit and dissemination of scientific research documents, whether they are published or not. The documents may come from teaching and research institutions in France or abroad, or from public or private research centers.
L'archive ouverte pluridisciplinaire HAL, est destinée au dépôt et à la diffusion de documents scientifiques de niveau recherche, publiés ou non, émanant des établissements d'enseignement et de recherche français ou étrangers, des laboratoires publics ou privés. 


\title{
$16^{\mathrm{TH}}$ INTERNATIONAL DEPENDENCY AND STRUCTURE MODELLING CONFERENCE, DSM 2014
}

PARIS, FRANCE, JULY 2 - 4, 2014

\section{Optimal Capacity Allocation for a Failure Resilient Electrical Infrastructure}

\author{
Yi-Ping Fang ${ }^{1}$, Nicola Pedroni ${ }^{1}$, Enrico Zio ${ }^{1,2}$ \\ ${ }^{1}$ Chair on Systems Science and the Energetic challenge, École Centrale Paris and \\ Supélec, France \\ ${ }^{2}$ Energy Department, Politecnico di Milano, Italy
}

\begin{abstract}
In this study, we tackle the problem of searching for the most favorable pattern of link capacities that makes a power transmission network resilient to cascading failures with limited investment costs. This problem is formulated within a combinatorial multi-objective optimization framework and tackled by evolutionary algorithms. A power flow model (namely, the ORNL-Pserc-Alaska (OPA) model) is embraced to simulate cascading failures in a network and to quantify its resilience. The framework of capacity allocation optimization is originally applied to the $400 \mathrm{kV}$ French power transmission network for the purpose of exemplification. The results show that cascade resilient networks tend to have a non-linear capacity-load relation: in particular, heavily loaded components have smaller unoccupied portion of capacity, whereas lightly loaded links present larger unoccupied portion of capacity which is in contrast with the linear capacity-load relation hypothesized in previous works.
\end{abstract}

Keywords: power transmission network, cascading failures, power flow model, capacity optimization, evolutionary algorithm

\section{Introduction}

Our modern society has come to depend on large-scale critical infrastructures (CIs) to deliver resources to consumers and businesses in an efficient manner. These CIs are complex networks of interconnected functional and structural elements. Large scale outages on these real-world complex networks, although infrequent, are increasingly disastrous to society, with estimates of direct costs up to billions of dollars and inestimable indirect costs. Typical examples include blackouts in power transmission networks (USCA, 2004, UCTE, 2007 and Pidd, 2012), financial bankruptcy (Battiston et al., 2007), telecommunication outages (Newman et al., 2002), and catastrophic failures in socio-economic systems (Zhao et al., 2011 and Kempe et al., 2003).

Research regarding modelling, prediction and mitigation of cascading failures in CIs, whereby small initial disturbances may propagate through the whole infrastructure system, has addressed the problem in different ways, including physical models for describing cascading failure phenomena (Dobson et al., 2001), control and defense strategies against cascading failures (Motter, 2004, Li et al., 2013 and Fang et al. 2013), analytical calculation of capacity parameters (Zhao et al., 2004), and modelling of the real-world data (Kinney et al., 2005).

In particular, various problems concerning the robustness and functionality of CI systems (ranging from power outages and Internet congestion to affordability of public transportation) are ultimately determined by the extent to which the CI capability 
matches supply and demand under realistic conditions (Kim and Motter, 2008). Continuous effort has been made to model the capacity-load relationship of realistic systems as well as to enhance the CI performance while limiting its cost. A homogeneous capacity-load relationship model is widely used in studying of CIs (Motter, 2004, Li et al., 2013, Fang et al. 2013 and Zio and Sansavini, 2011), whereby the capacity of a link (node) is assumed to be proportional to its initial flow. However, it has been argued that this is unrealistic and empirical data suggests that the relationship between capacity and load of transmission lines is non-linear; heavily loaded lines usually have a lower tolerance parameter than lightly loaded lines (Kim and Motter, 2008). Most recently, a two-step function (non-linear) was proposed for the relationship between vertices capacity and load (Wang and Kim, 2007). Although over-simplified, it has been shown to be efficient to prevent cascade by protecting highest-load vertices. It is shown that a more complex heuristic capacity model, whereby vertices with both higher loads and larger degrees are paid more extra capacities, can achieve better network robustness than previous models under the same amount of available resources (Li et al., 2008).

In the present study, we tackle this issue from a more systematical perspective by searching for the most favorable strategy of capacity allocation for a power transmission network in resisting to cascading failures, while keeping the total resource (capacity) limited (i.e., while minimizing the network cost). The problem is formulated within a large-scale, nonlinear and combinatorial multi-objective optimization framework and is solved by a fast and elitist genetic algorithm, namely NSGA-II (Deb et al., 2002). For illustration, we apply the method to a realistic electrical infrastructure, i.e., the $400 \mathrm{kV}$ French power transmission network.

The reminder of this paper is organized as follows. We formulate the multi-objective optimization model taking investment cost and failure resilience into account in Section 2. Section 3 briefly introduces the procedure of the NSGA-II algorithm. Section 4 illustrates the French $400 \mathrm{kV}$ power transmission network case study and the results analysis. Conclusion are drawn on the Section 5.

\section{Formulation of the Multi-objective Optimization Problem}

\subsection{The ORNL-Pserc-Alaska (OPA) Cascading Failure Model}

The ORNL-Pserc-Alaska (OPA) model has been proposed by researchers at Oak Ridge National Laboratory (ORNL), Power System Engineering Research Center of Wisconsin University (PSerc), and Alaska University (Alaska) (Dobson et al., 2001, Carreras et al., 2004). The OPA model is built upon the Self-Organized Criticality (SOC) theory, contains two different time scale dynamics, i.e., fast power flow dispatching dynamics and slow power grid growth dynamics, and describes the complexity and criticality of power systems. It is a novel and powerful tool for analyzing power systems. Our analysis focuses on the fast power flow dynamics.

The cascading failure model is based on the standard DC power flow equation,

$$
F=A \cdot P
$$

where $F$ is a vector whose $M$ components are the power flows through all the lines in the network, $F_{l}(l \in E), P$ is a vector whose $N-1$ components are the power injection of each 
node, $P_{i}(i \in V)$ ( $E$ is the set of links, $V$ is the set of nodes, and $N$ is the total number of nodes in the network, $N=|V|$ ), with the exception of the reference generator, $P_{0}$, and $A$ is a constant matrix that depends on the network structure and impedances (Dobson et al., 2001). The reference generator power is not included in the vector $P$ to avoid singularity of $A$ as a consequence of the overall power balance.

The generator power dispatch is solved using standard linear programming methods. Using the input power demand, the power flow equation (1) is solved with the condition of minimizing the following cost function:

$$
f=\sum_{i \in V_{G}} P_{i}(t)+K \sum_{j \in V_{D}} P_{j}(t)
$$

This definition gives preference to generation shift whilst assigning a high cost (set $K=$ 100) to load shedding and it is assumed that all generators operate at the same cost and that all loads are served with equal priority. The minimization is done with the following constraints:

(1) Generator power injections are generally positive and limited by installed capacity limits: $0 \leq P_{i} \leq P_{i}^{\max }, i \in V_{G}$.

(2) Loads always have negative power injections: $P_{j}^{d e m} \leq P_{j} \leq 0, j \in V_{D}$.

(3) The flow though links is limited by link capacities: $\left|F_{l}\right| \leq C_{l}, l \in E$.

(4) Total power generation and consumption remain balanced: $\sum_{i \in V_{G} \cup V_{D}} P_{i}=0$.

After solving the linear optimization by using the simplex (Flannery et al., 1992), we examine which lines are overloaded. A line is considered to be overloaded if the power flow through it is within $1 \%$ of the limit capacity $C_{l}$. Each overloaded line may outage with probability $p_{1}$ ( $p_{1}$ is set as 1 in the case study to ensure its comparability with ML). If an overloaded line experiences an outage, its power flow limit $C_{l}$ is divided by a very large number $k_{1}$ to ensure that practically no power may flow through the line. Besides, to avoid a matrix singularity from the line outage, the impedances of failed lines are multiplied by a large number $k_{2}$, resulting in changes of the network matrix A.

Load shedding is utilized to quantify the damage of the cascading failure. For an individual node, load shedding is defined as absolute value of the difference between its power injection and demand:

$$
L S_{j}=\left|P_{j}^{d e m}-P_{j}\right|, j \in V_{D}
$$

Subsequently, total load shedding for the system is:

$$
L S=\sum_{j \in V_{D}} L S_{j}
$$

Finally, system load shedding is normalized by its total demand $D$ and used as a measure of damage to the system resulting from a cascading failure:

$$
\overline{L S}=\frac{L S}{D}=\frac{\sum_{j \in V_{D}} L S_{j}}{\sum_{j \in V_{D}} P_{j}^{d e m}}
$$

The OPA model seeks to faithfully describe the dispatching dynamics of the power flows during the evolution of the failure propagation following the initial disturbances, by explicitly incorporating the standard DC power flow equations and minimizing generation cost and load shedding. The fact that simulation results from OPA model are consistent with historical blackout data for real power systems has justified its effectiveness (Carreras et al., 2004). 


\subsection{Optimization Formulation}

In this section, we generally frame the problem of searching the most favorable pattern of link capacities in a realistic power transmission network, so as to optimize its resilience against cascading failures. By associating a cost to (the capacity of) each link of the network, the optimization process also seeks to minimize the total cost.

Specifically, we define the variables to be optimized as the capacities of the links in the network $C_{l}, l \in E$. Any non-negative vector $C \in \boldsymbol{R}_{+}^{M}$ could represent a potential solution. It is noted that the searching space $\boldsymbol{R}_{+}^{M}$ is intractably large in reality, where a power transmission network usually has hundreds of thousands of links.

We then assume that the cost associated with each link capacity is linearly proportional to the value of the capacity, with coefficient $\varphi$ (we simply set $\varphi$ as 1 in our case study). The total investment cost related to a capacity allocation pattern $C \in \boldsymbol{R}_{+}^{M}$ in the power transmission network can then be defined as

$$
\operatorname{Cost}(C)=\sum_{l \in E} \varphi C_{l}
$$

The network damage resulting from a cascading failure in the presence of a given capacity pattern can be obtained by running the OPA simulation in correspondence of the capacity pattern and then using Equation (5). The cascade is initiated by the failure of a single link in each model. The single link is randomly selected from the set of links $E$ in the network with equal probability. Then, the algorithms for cascading simulation proposed in Section 2.1 are applied. The cascade simulations run over several iterations until they either converge or exceed the maximum number of steps (we use maximum 20 iterations in the case study). Finally, the network vulnerability for a given capacity allocation pattern $C$ is obtained as the average system load shedding over various random triggers (we use $T=30$ triggers in the case study):

$$
\operatorname{Vul}(C)=\frac{1}{T} \sum_{t=1, \ldots, T} \overline{L S}^{t}(C) .
$$

Through the quantification of the capacity allocation cost and cascading failure vulnerability, the capacity allocation problem is formulated as a multi-objective optimization:

$$
\left\{\begin{array}{l}
\min _{C \in R_{+}^{M}} \operatorname{Cost}(C) \\
\min _{C \in R_{+}^{M}} \operatorname{Vul}(C)
\end{array}\right.
$$

Observe that under this definition the most cascade-resilient network might be the network with infinite capacity, which obviously would conflict with the objective of minimizing cost.

\section{Multi-objective Evolutionary Algorithms (MOEAs) for Optimal Capacity Allocation}

Multi-objective evolutionary algorithms (MOEAs) have proven be to general, robust and powerful search tools that are desirable for tackling problems involving i) multiple conflicting objectives, and ii) intractably large and highly complex search spaces (Zitzler et al., 2004). In extreme synthesis, the main properties of Evolutionary Algorithms (EAs) are that the search for the optima is conducted (i) using a (possibly) large population of multiple solution points or candidates, (ii) using operations inspired by the evolution of 
species, such as breeding and genetic mutation, (iii) using probabilistic operations and (iv) using information on the objective or search functions and not on its derivatives. The main advantages are: (i) fast convergence to near global optima, (ii) superior global searching capability in complicated search spaces and (iii) applicability even when gradient information is not readily achievable. MOEAs rely on the following concepts (Deb et al., 2002):

(1) Pareto front: The locus that is formed by a set of solutions that are equally good when compared to other solutions of that set is called Pareto front.

(2) Non-Domination: Non-dominated or Pareto-optimal solutions are those solutions in the set which do not dominate each other, i.e., neither of them is better than the other in all the objective function evaluations. The solutions on each Pareto front are Pareto-optimal with respect to each other.

In this study, we use a fast and elitist genetic algorithm, namely, NSGA-II (Deb et al., 2002), to solve the multi-objective optimization problem (9). NSGA-II has been proved to be an efficient algorithm to find Pareto optimal solutions (Konak et al., 2006); for further details about this algorithm and relevant surveys on multi-objective evolutionary optimization, the reader is referred to Deb et al. (2002), Konak et al. (2006) and Zitzler et al. (2004). The complete procedure for our capacity allocation optimization problem is detailed as follows:

step 1. Read power transmission network data (line, bus, adjacency matrix, etc.) and fix the MOEA parameters (i.e., population size, maximum generation, etc.);

step 2. Randomly initialize a (parent) population of possible solutions (individuals) and evaluate the fitness of each individual with respect to the two objective functions in Formula (9); sort the parent population according to the nondomination criterion (Deb et al., 2002);

step 3. Select the parents which are fitter for reproduction by using a binary tournament selection (Deb et al., 2002); the procedure is said that fitter individuals are selected with a higher probability;

step 4. Generate an offspring population by crossover and mutation operators, and evaluate the fitness of each individual in the offspring population with respect to the two objective functions in Formula (9).

step 5. Combine the parent and offspring populations to generate a new "trial" aggregate population and perform non-dominated sorting on the "trial" population;

step 6. Generate a new parent population by selecting the best solutions in the sorted "trial" population, until a desired population size is reached;

step 7. If the stop condition is met, then terminate the iteration; otherwise, go to step 3.

The non-dominated solutions of the last population constitute the Pareto optimal front of the optimization problem at hand.

\section{Case Study and Results Analysis}

In this paper, the $400 \mathrm{kV}$ French power transmission network (Figure 1) is taken for exemplification of the proposed approach. The network is built from the data on the 400 
$\mathrm{kV}$ transmission lines of the RTE website (RTE, 2011). It has 171 nodes (substations) and 220 edges (transmission lines). We distinguish the generators, which are the source of power, from the other distribution substations, that receive power and transmit it to other substations or distribute it in local distribution grids. By obtaining the power plants list from the EDF website (EDF, 2013) and relating them with the ID of the buses in the transmission network, we have 26 generators and 145 distributors. Only the nuclear power plants, hydraulic plants and thermal power plants whose installed capacities are larger than $1000 \mathrm{MW}$, are considered.

For optimal allocation of link capacity in the network, the NSGA-II algorithm introduced in Section 4 is applied with regarding to the objectives of minimizing cascade vulnerability and investment cost, expressed by Formula (9). The parameters values used in the NSGA-II algorithm are reported in Table 1.

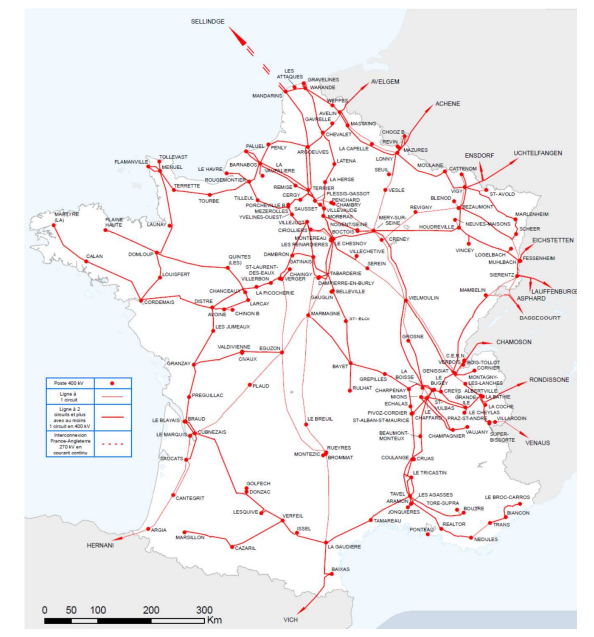

Figure 1. The $400 \mathrm{kV}$ French power transmission network

Table 1. Parameters of NSGA-II algorithm

\begin{tabular}{|c|c|}
\hline Parameters & Values \\
\hline Population size & 80 \\
\hline Maximum generation & 1500 \\
\hline Crossover probability & 0.9 \\
\hline Mutation probability & 0.1 \\
\hline Crossover operator & 20 \\
\hline Mutation operator & 20 \\
\hline
\end{tabular}

The Pareto dominance front obtained by the NSGA-II algorithm for our capacity allocation problem at convergence is illustrated in Figure 2 (triangles). The result obtained by employing a homogeneous capacity allocation strategy is also reported here (solid line) for comparison. In the classic homogeneous allocation strategy, the capacity of link $l$ is assumed to be proportional to its initial flow with a network tolerance 
parameter $\alpha$, i.e., $C_{l}=\alpha F_{l}(0)$. The concept of tolerance parameter can be understood as an operating margin allowing safe operation of the component under potential load increment. Besides, the cost of each capacity (allocation pattern) proposed by the optimization algorithm is normalized by the corresponding total initial network flow ${ }^{1}$ to facilitate the comparison of our results and indicated as $\overline{\operatorname{Cost}}$. It can be seen that the multi-objective optimization approach produces better solutions: actually, the Pareto front is closer to the coordinate axes. This is evident that the linear (homogeneous) capacity-load relationship is not optimal for a cost-efficient and cascade-resilient network.

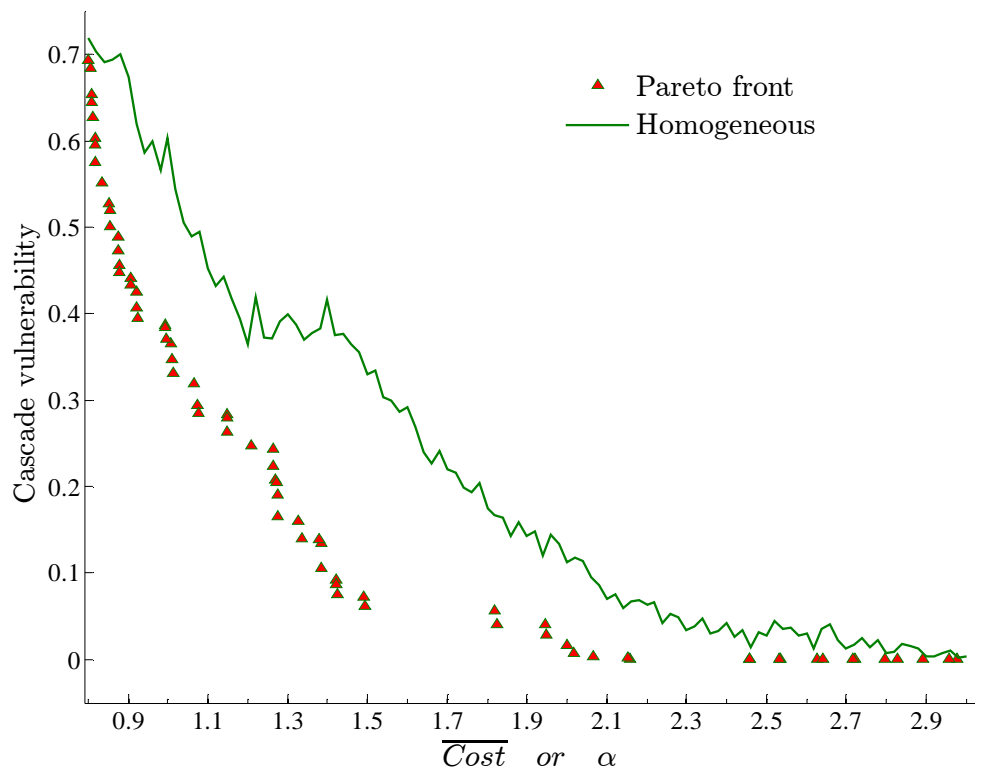

Figure 2. Pareto front and obtained in the multi-objective optimization framework of Section 2 (triangles) together with the results obtained by employing a homogeneous capacity allocation strategy (solid line)

We then analyze what the patterns of link capacities look like in the optimized solutions, especially when lower network cascade vulnerability (higher network resilience) is demanded, i.e., which type of capacity allocation pattern is the most favorable in resisting to cascading failure. We tackle this problem by investigating the "expected" network link capacity pattern as a function of cascade vulnerability, i.e., the configuration of capacity pattern "averaged" over all possible solutions of the Pareto front lying within a given "regime" (i.e., interval) of cascade vulnerability of interest. Parameter $\beta^{s}$ is used to represent the "regime" of cascade vulnerability, where $s$ indicates the size of the corresponding interval. It is noted that smaller $\beta^{s}$ represents higher network resilience.

\footnotetext{
${ }^{1} \mathrm{By}$ this definition, the normalized cost $\overline{\operatorname{Cost}}$ has precisely the same physical meaning with the network tolerance parameter $\alpha$.
}

DSM 2014 
Part 0: Basic instructions

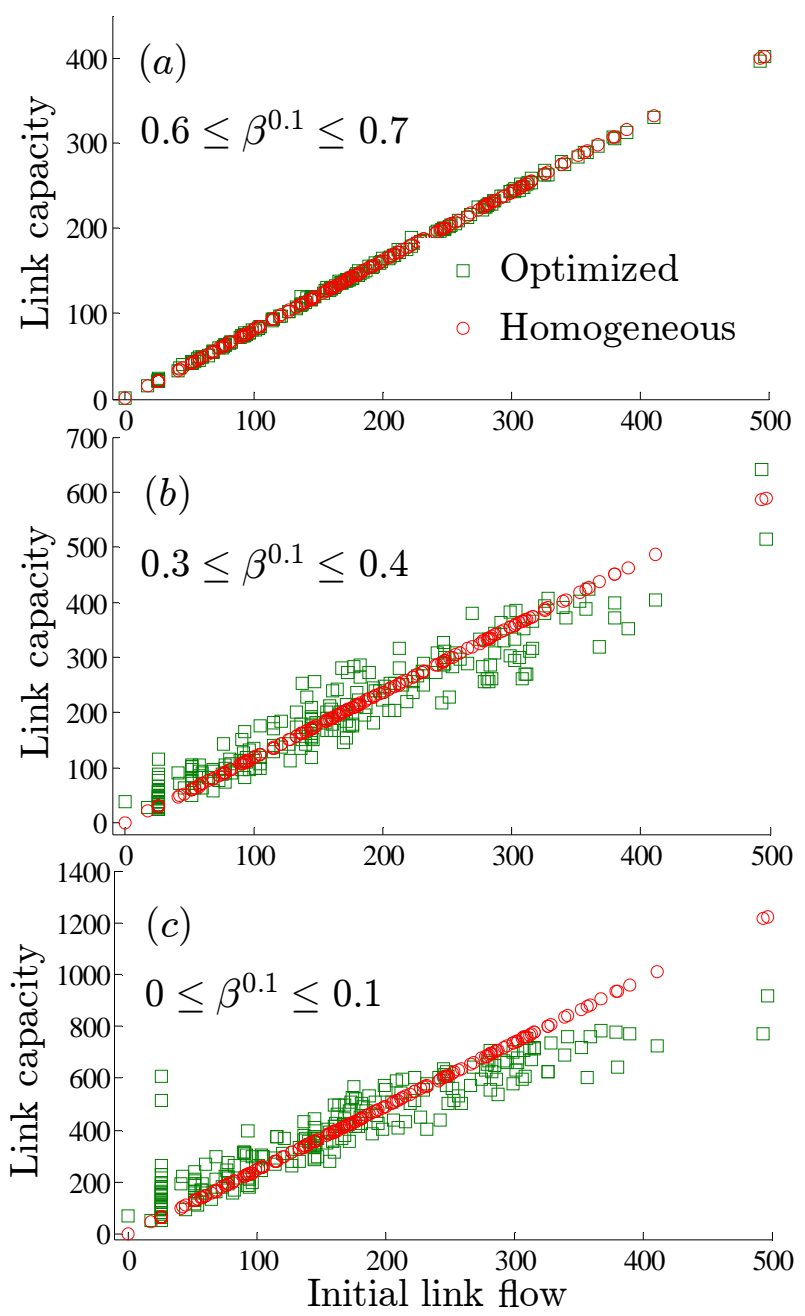

Figure 3. "Averaged" optimal link capacity patterns for three different levels of cascade vulnerability $\left(0.6 \leq \beta^{s} \leq 0.7,0.3 \leq \beta^{s} \leq 0.4,0 \leq \beta^{s} \leq 0.1\right)$ in the Pareto front. The scatter plot shows the relationship between the link capacities and the initial link flows in a homogeneous allocation strategy, where the capacity of a link is assumed to be proportional to its initial flow (circles) and after in the optimization-based approach of Section 3(squares).

Figure 3 reports the results of averaged link capacities patterns for three different levels of cascade vulnerability, i.e., $0.6 \leq \beta^{s} \leq 0.7,0.3 \leq \beta^{s} \leq 0.4$ and $0 \leq \beta^{s} \leq 0.1$ in the case of a homogeneous allocation strategy (circles) and of the optimization-based approach in our study (squares). It is found that the optimal link capacity patterns exhibit interesting characteristics. The optimal link capacities patterns are similar to their corresponding homogeneous allocations only in less resilient networks, (i.e., when $0.6 \leq \beta^{s} \leq 0.7$ ), where the objective of minimizing investment cost is much more 
biased (Figure 3a). When we increase the importance of minimizing the network vulnerability (e.g., for $0.3 \leq \beta^{s} \leq 0.4$ and $0 \leq \beta^{s} \leq 0.1$ ), the optimal link capacities show a non-linear relationship with respect to their initial flows, as shown in Figure 3(b) and Figure 3(c). Specifically, the heavily loaded links tend to decrease their capacities and the lightly loaded links tend to increase their capacities. That is to say, the unoccupied portion of capacity tends to decrease in links with larger loads, and the unoccupied portion of capacity tends to increase in the less loaded links. Furthermore, the higher the importance given to the minimization of network cascade vulnerability, the more pronounced the non-linear behavior is, as shown in Figure 3(b) and Figure 3(c). Our findings are consistent with the empirical observations and results from the traffic fluctuation model (Kim and Motter, 2008).

\section{Conclusion}

In this paper, we have tackled the problem of searching the most favorable pattern of link capacity allocation for a CI network with the objective of resisting to cascading failures with limited investment costs. This problem has been formulated within a multiobjective optimization framework and has been solved by an evolutionary algorithm, namely the NSGA-II. The optimization has been carried out using a physical power flow cascading failure model. The 400kV French power transmission network has been taken as a case study for illustration.

Our optimization results produce improved Pareto solutions with respect to those obtained by assuming a classical homogeneous allocation strategy. In addition, the optimal link capacity allocations show a non-linear capacity-load relation: the unoccupied portion of capacity tends to decrease in links with larger loads, whereas the unoccupied portion of capacity tends to increase in the lightly loaded links. This is in sharp contrast with the linear capacity-load relation hypothesized in previous works (Motter, 2004, Li et al., 2013, Fang et al. 2013 and Zio and Sansavini, 2011). This nonlinear behavior is probably a consequence of the following observation: since larger loads in heavily loaded components tend to result from a large number of flow events, the relative size of the fluctuations in these components tends to be small when other lightly loaded components fail during a cascading failure; considering that the unoccupied capacity is the operating margin that allow safe operation for the component under potential load increment (and is mainly determined by the perturbations caused by the failure of other components of the network), this explains why in the optimal solutions the unoccupied capacity tends to be smaller for links with larger loads.

\section{References}

Battiston, S., Delli Gatti, D., Gallegati, M., Greenwald, B., \& Stiglitz, J. E., 2007. Credit chains and bankruptcy propagation in production networks. Journal of Economic Dynamics and Control 31(6), 2061-2084.

Carreras B. A., Newman D. E., Dobson I., and Poole A. B., 2004. Evidence for self-organized criticality in a time series of electric power system blackouts, Circuits and Systems I: Regular Papers, IEEE Transactions on 51(9): 1733-1740.

Deb K., Pratap A., Agarwal S., and Meyarivan T., 2002. A fast and elitist multi-objective genetic algorithm: Nsga-ii, Evolutionary Computation, IEEE Transactions on 6(2): 182-197.

DSM 2014 
Deb K., Pratap A., Agarwal S., and Meyarivan T., 2002. A fast and elitist multi-objective genetic algorithm: Nsga-ii, Evolutionary Computation, IEEE Transactions on 6(2): 182-197.

Dobson I., Carreras B. A., Lynch V. E., Newman D. E., 2001. An initial model for complex dynamics in electric power system blackouts, in: Proceedings of Annual Hawaii International Conference on System Sciences, pp. 51-51.

EDF, 2013. En direct de nos centrales. Retrieved Avril 2013, http://france.edf.com/france45634.html.

Fang Y.-P., Pedroni N., and Zio E., 2013. Optimal production facility allocation for failure resilient critical infrastructures, in: ESREL 2013.

Flannery B. P., Press W. H., Teukolsky S. A., and Vetterling W., 1992. Numerical recipes in c, Press Syndicate of the University of Cambridge, New York.

Kempe, D., Kleinberg, J., and Tardos, É., 2003. Maximizing the spread of influence through a social network. In Proceedings of the ninth ACM SIGKDD international conference on Knowledge discovery and data mining ACM: 137-146.

Kim D.-H., and Motter A. E., 2008. Fluctuation-driven capacity distribution in complex networks, New Journal of Physics 10(5), 053022.

Kinney R., Crucitti P., Albert R., and Latora V., 2005. Modeling cascading failures in the north american power grid, The European Physical Journal B-Condensed Matter and Complex Systems 46 (1), 101-107.

Konak A., Coit D. W., and Smith A. E., 2006. Multi-objective optimization using genetic algorithms: A tutorial, Reliability Engineering \& System Safety 91(9): 992-1007.

Li P., Wang B.-H., Sun H., Gao P., and Zhou T., 2008. A limited resource model of fault-tolerant capability against cascading failure of complex network, The European Physical Journal B 62(1): 101-104.

Li, Y. F., Sansavini, G., and Zio, E., 2013. Non-Dominated Sorting Binary Differential Evolution for the Multi-Objective Optimization of Cascading Failures Protection in Complex Networks. Reliability Engineering \& System Safety 111: 195-205.

Motter, A. E., 2004. Cascade control and defense in complex networks. Physical Review Letters 93(9): 098701.

Newman, M. E., Forrest, S., and Balthrop, J., 2002. Email networks and the spread of computer viruses. Physical Review E 66(3), 035101.

Pidd H., 2012. India blackouts leave 700 million without power. The Guardian 31 July 2012.

RTE, 2011. Le Réseau de Transport d'Electricité $400 \mathrm{kV}$. http://www.rte-france.com.

UCTE, 2007. Final Report System Disturbance on 4 Nov. 2006, Union for the Coordination of Transmission of Electricity, Tech. Rep.

USCA, 2004. Final Report on the August 14, 2003 Blackout in the United States and Canada, USCanada Power System Outrage Task Force, Tech. Rep.

Wang B., Kim B. J., 2007. A high-robustness and low-cost model for cascading failures, EPL (Europhysics Letters) 78(4), 48001.

Zhao L., Park K., Lai Y.-C., 2004. Attack vulnerability of scale-free networks due to cascading breakdown, Physical review E 70(3), 035101.

Zhao, K., Kumar, A., Harrison, T. P., and Yen, J., 2011. Analyzing the resilience of complex supply network topologies against random and targeted disruptions. Systems Journal, IEEE 5(1), 28-39.

Zio E., and Sansavini G., 2011. Modeling interdependent network systems for identifying cascadesafe operating margins, Reliability, IEEE Transactions on 60(1): 94-101.

Zitzler E., Laumanns M., and Bleuler S., 2004. A tutorial on evolutionary multiobjective optimization, in: Metaheuristics for Multiobjective Optimisation, Springer, pp. 3-37.

Contact: Y.-P. Fang, chair on Systems Science and the Energetic challenge, École Centrale Paris and Supélec, Grande Voie des Vignes, 92290, Châtenay-Malabry, France, yiping.fang@ecp.fr, http://www.lgi.ecp.fr/ li/yiping_fang.html. 